\title{
Circulación de animales en Iberia durante la Prehistoria reciente y la época clásica: métodos de análisis, primeros datos y retos de futuro
}

\author{
Animal mobility in Iberia during Late Prehistory and \\ Classical times: analytical methods, first data, \\ and challenges for the near future
}

\author{
Silvia VALENZUELA LAMAS \\ Institució Milà i Fontanals (CISC). Archaeology of Social Dynamics (ASD) \\ C/ Egipcíaques, 15, E-08001 Barcelona \\ svalenzuela@imf.csic.es
}

\begin{abstract}
El estudio de la movilidad se ha convertido en un tema clave de investigación en arqueología en los últimos años. El desarrollo de los estudios químicos (química isotópica, ADN) y de morfometría geométrica y, sobre todo, su aplicación en arqueología, han permitido obtener datos de primera mano sobre muestras humanas y animales del pasado. Este artículo discute la potencialidad y las limitaciones de las técnicas más utilizadas (morfometría geométrica, ADN antiguo, isótopos de estroncio y oxígeno) y ofrece una primera síntesis de datos de movilidad animal durante la Prehistoria reciente y la época clásica en la península ibérica y el Reino Unido.
\end{abstract}

\section{PALABRAS CLAVE}

ARQUEOZOOLOGÍA, MOVILIDAD, QUÍMICA ISOTÓPICA, MORFOMETRÍA GEOMÉTRICA, ADN ANTIGUO

The study of mobility has become a key research topic in Archeology in recent years. The development of geometric morphometrics and chemical studies (e.g. isotopic chemistry, DNA), and their application in archeology, produces first-hand data on human and animal samples from the past. This article discusses the potential and limitations of the most used techniques (geometric morphometrics, ancient DNA, strontium and oxygen isotopes), and offers a first synthesis of references and data about animal mobility during Late Prehistory and Classical times in the Iberian Peninsula and the United Kingdom.

\section{KEYWORDS}

ARCHAEOZOOLOGY, MOBILITY, ISOTOPIC CHEMISTRY, GEOMETRIC MORPHOMETRICS, ANCIENT DNA 


\section{Introducción}

El estudio de la movilidad humana y de las áreas de captación de recursos son temas recurrentes en la investigación sobre el pasado, y han sido estudiados desde muchos puntos de vista y con metodologías distintas. El estudio de la movilidad animal, sin embargo, ha sido mucho más limitado, a veces reducido a la presencia de nuevas especies o a la importación de especies exóticas (p. ej., Morales et al., 1995a; 1995b; Riquelme-Cantal et al., 2008).

El desarrollo de los estudios de ADN y de química isotópica de los últimos veinte años ha cambiado este panorama $y$, actualmente, los estudios sobre movilidad animal se han multiplicado en arqueología (p. ej., Balasse et al., 2002; Viner et al., 2010; Makarewicz y Sealy, 2015; Gerling et al., 2017; Verdugo et al., 2019). Esto posibilita relacionar y comparar los datos de movilidad animal con los de movilidad humana, así como con otros indicadores (sistemas políticos, grado de integración económica, funcionalidad de los yacimientos, etcétera; ver Kristiansen, 2014, como ejemplo). Aun así, actualmente son muchas las lagunas temporales y geográficas, tanto en la península ibérica como en el resto del mundo. En el caso de las especies domésticas, la situación se agrava por el hecho de que diferentes especies pueden tener distintos patrones de movilidad.

Este artículo pretende ofrecer algunos datos disponibles sobre los patrones de movilidad animal en la península ibérica y en el Reino Unido — uno de los territorios donde hay más datos publicados-y, sobre todo, exponer las limitaciones metodológicas a las que nos enfrentamos y los retos que habrá que abordar en un futuro próximo.

\section{Muestreo, conservación y límites de unas disciplinas en desarrollo}

El primer reto al que se enfrenta cualquier especialista es el muestreo y la decisión de qué técnicas aplicar: ¿Qué piezas hay que seleccionar? ¿Cuál es el número mínimo de muestras necesario? ¿Cuánto cuestan los análisis? La respuesta a estas preguntas es muy variable y depende de las preguntas de investigación. Así mismo, también depende del laboratorio, de la técnica utilizada y del grado de preparación y limpieza que necesita cada analítica. El rango puede ir desde cubrir exclusivamente la mano de obra (p. ej., morfometría geométrica) hasta cubrir el coste de las largas horas de trabajo y la manipulación de los productos químicos en un ambiente completamente estéril, dentro de un laboratorio especializado (caso de las muestras de ADN antiguo).

En líneas generales, para la mayoría de análisis isotópicos y morfométricos la mejor pieza anatómica son los dientes (p. ej., Sponheimer y Lee-Thorp, 1999; Balasse et al., 2002; Viner et al., 2010; Nuviala et al., 2014; Duval et al., 2015; Evin et al., 2017). En el caso de la morfometría geométrica (GMM, por sus siglas en inglés) y los análisis de ADN, otros elementos anatómicos utilizados son los metacarpos y metatarsos (p. ej., Schlumbaum et 
al., 2006; Davis et al., 2012; Colominas et al., 2015) y, en GMM, también los astrágalos (p. ej., Colominas et al., 2019; Pöllath et al., 2019).

\subsection{Una guía rápida en la selección de materiales y técnicas}

Todas las técnicas arriba mencionadas (GMM, isótopos de estroncio y oxígeno, ADN) tienen un gran potencial en los estudios de movilidad, pero también sus limitaciones. Con el objetivo de presentar una guía en la toma de decisiones, en la tabla 1 sintetizamos las características principales de cada una de ellas, los elementos anatómicos a muestrear, y el número mínimo de muestras requerido. De todas maneras, son las preguntas de investigación, la disponibilidad de los materiales y el presupuesto los que marcarán la decisión final en cada caso.

De cara a la conservación de los materiales, la morfometría geométrica es la técnica más económica y la única que no es destructiva. La base metodológica principal es que la forma de un elemento anatómico (entendida como la combinación entre tamaño y conformación) refleja características fenotípicas y se puede describir geométricamente a partir de puntos de referencia y de contorno (Bookstein, 1991; Rohlf y Marcus, 1993). Así, es posible reconocer diferentes morfotipos y evaluar el grado de especificidad de una forma concreta. En otras palabras, la morfometría geométrica permite describir la forma con una

Tabla 1. Guía con las principales características de las técnicas mencionadas en el artículo

\begin{tabular}{|c|c|c|c|c|}
\hline Técnica & Destructiva? & Elementos de muestreo & $\begin{array}{l}\text { Número mínimo } \\
\text { de elementos }\end{array}$ & Comentarios \\
\hline Morfometría geométrica & No & $\begin{array}{l}\text { m2, m3, astrágalo, } \\
\text { metacarpo, metatarso }\end{array}$ & 30 iguales & $\begin{array}{l}\text { Se pueden mezclar elementos } \\
\text { derechos e izquierdos pero no } \\
\text { elementos distintos (una M2 inferior } \\
\text { es distinta de una M2 superior) }\end{array}$ \\
\hline ADN & Sí & $\begin{array}{l}\text { dientes, huesos con cortical } \\
\text { espesa (p.ej: metacarpo, } \\
\text { metatarso) }\end{array}$ & ver comentarios & $\begin{array}{l}\text { La buena conservación del colágeno } \\
\text { es fundamental. Hay que evitar los } \\
\text { huesos esponjosos (costillas, epífisis } \\
\text { de huesos largos). En la península } \\
\text { ibérica, en yacimientos al aire libre, } \\
\text { suelen funcionar un tercio de las } \\
\text { muestras }\end{array}$ \\
\hline Isótopos de estroncio & Sí & dientes & 10 & $\begin{array}{l}\text { En estudios de movilidad estacional } \\
\text { hay que contar un mínimo de } 3 \\
\text { muestras por diente }\end{array}$ \\
\hline Isótopos de oxígeno & Sí & dientes & 10 & $\begin{array}{l}\text { En estudios de movilidad estacional } \\
\text { hay que contar un mínimo de } 5 \\
\text { muestras por diente }\end{array}$ \\
\hline
\end{tabular}



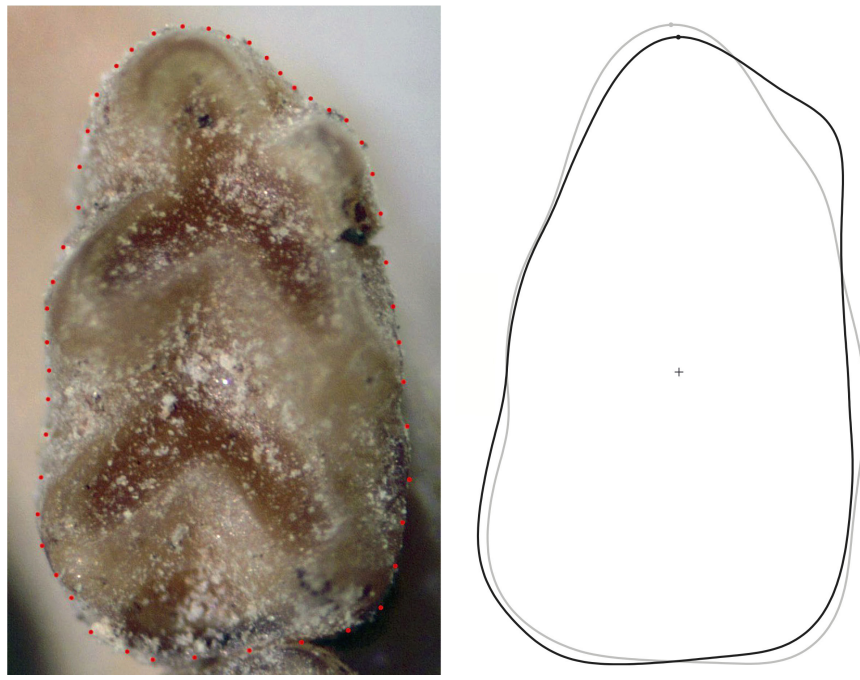

Figura 1. Ejemplo de fotografía de diente y digitalización (izquierda) y perfiles reconstruidos de dos especies de ratones ( $M$. musculus y $M$. spretus).

precisión que no es posible alcanzar con la morfología clásica y evaluar si la población analizada tiene una morfología muy específica (de astrágalo, de tercer molar, etc.) o bien se trata de una población que no se distingue de otras. En este sentido, es preciso tener un número de muestras suficientemente grande para poder determinar si hay diferencias significativas entre poblaciones, y comprobar que la falta de diferencias no se debe a un número bajo de efectivos que no permite discriminar una población de otra. Así, si dos poblaciones presentan diferencias significativas es que, muy probablemente, se trata de dos poblaciones diferentes. En cambio, dos poblaciones originariamente diferentes podrían arrojar diferencias no significativas debido a un bajo número de efectivos.

La forma se puede analizar en dos y en tres dimensiones (2D y 3D). Para los análisis en $2 \mathrm{D}$ es preciso tomar fotografías perpendiculares al elemento que se está analizando y siempre a la misma escala (figura 1). Posteriormente, se determinan los elementos preeminentes (p. ej., contorno, puntos de referencia) y se analizan específicamente para caracterizar la forma y la existencia de diferencias entre poblaciones (p. ej., Macholán, 2006; ValenzuelaLamas et al., 2011; Evin et al., 2015). En el caso de los análisis 3D, es preciso medir el volumen del objeto, generalmente con un escáner o con fotogrametría. En arqueología hay ejemplos sobre industria lítica (p. ej., Lycett et al., 2010) y cerámica (p. ej., Selden et al., 2014) y en arqueozoología se ha aplicado sobre cráneos de suido (Owen et al., 2014).

La GMM es actualmente un campo en expansión. Su limitación principal radica en el elevado número de restos que requiere. Se pudiera pensar que es fácil conseguir un mínimo de 30 elementos iguales de una especie, pero la experiencia nos dice que solo los conjuntos grandes (NRD>300) permiten disponer de suficientes elementos de una misma fase cronológica (p. ej., astrágalos o terceros molares inferiores). Esto se agrava en el caso de especies poco representadas en el registro. 
El análisis de los isótopos de estroncio $\left({ }^{87} \mathrm{Sr} /{ }^{86} \mathrm{Sr}\right)$ no suele presentar problemas de conservación y es, probablemente, la técnica más precisa para determinar movimientos de animales entre zonas geológicas diferentes. En el caso de los herbívoros, la base metodológica es que la ratio entre el isótopo ${ }^{87} \mathrm{Sr}$ y el ${ }^{86} \mathrm{Sr}$ depende de la geología en la que pastan y abrevan los individuos. La ratio isotópica pasa del agua y los vegetales a los tejidos (huesos y dientes) casi sin fraccionamiento isotópico, por lo que es posible ver si a lo largo del período de mineralización de un diente ha habido cambios en la ratio isotópica, y también si el individuo tiene una ratio isotópica diferente a la de la geología en la que se encuentra el yacimiento (Bentley, 2006).

La principal limitación de esta técnica — aparte de que es destructiva, como todos los análisis químicos - es que no existen, por el momento, mapas de estroncio detallados para cada zona geográfica, y estudios recientes demuestran que hay solapamiento en las ratios de estroncio de substratos geológicos diferentes (Willmes et al., 2018). Así pues, es preciso muestrear no solo material arqueológico, sino también vegetación circundante no contaminada, ${ }^{1}$ para poder determinar la variabilidad local de estroncio de cada yacimiento. Otra limitación es la variabilidad geológica a escala local: hay zonas con una geología muy diversa, en las que la variabilidad potencial de estroncio en un rango de $50 \mathrm{~km}$ es muy amplia, mientras otras zonas son geológicamente muy homogéneas y, por tanto, se pueden producir movimientos sin que sea posible distinguirlos isotópicamente. Finalmente, son pocos los estudios (Britton et al., 2009; Towers et al., 2017) que han analizado cómo cambia la ratio isotópica de estroncio en animales de los cuales se conocen sus movimientos y su dieta en vida, por lo que se desconoce el ritmo de asimilación del estroncio y los posibles efectos de promedio debido a las técnicas de muestreo, que suelen incluir varias capas de mineralización (Hoppe et al., 2004; Towers et al., 2010).

Pese a estas limitaciones, es posible observar variaciones a lo largo de la corona dental de los dientes hipsodontos (p. ej., Hoppe et al., 2004; Bentley y Knipper, 2005; Towers et al., 2010; Viner et al., 2010). En este caso, es aconsejable tomar un mínimo de tres muestras por diente para poder observar si hay movimientos de «ida y vuelta» entre zonas geológicas diferentes.

En el caso de los isótopos de oxígeno $\left({ }^{18} \mathrm{O} /{ }^{16} \mathrm{O}\right)$, es posible analizar la relación isotópica bien sea del carbonato, bien del fosfato del esmalte dental. Los primeros estudios sobre material arqueológico favorecían el análisis de fosfato, por que el enlace químico P-O es mucho más fuerte que el del C-O, por lo que es más resistente a la diagénesis. Esto era de especial importancia en el análisis de huesos largos. Pero en los últimos años noventa se demostró que el esmalte dental, por su estructura cristalina y durabilidad, es fiable tanto para ratios isotópicas de oxígeno en carbonato como para fosfato (p. ej., Bocherens et al., 1996; Cerling et al., 1997; Sponheimer y Lee-Thorp, 1999), siendo el proceso químico en carbonato más sencillo comparado con el necesario en fosfato (Sponheimer y Lee-Thorp, 1999).

1. Las muestras vegetales más fiables son las hojas de árboles de raíces profundas (p. ej., encinas) tomadas fuera de los cauces de los ríos y zonas de deposición de sedimentos, y lejos (más de 100 m) de casas, industrias, carreteras y, en general, cualquier infraestructura que implique la deposición de aguas residuales o remoción de tierra. 
En cualquier caso, la relación isotópica de oxígeno $\left(\delta^{18} \mathrm{O}\right)$ está directamente relacionada con la del agua corporal, que a su vez refleja la ratio del agua ingerida en el momento de la formación del esmalte, previo fraccionamiento dependiendo de las influencias fisiológicas y metabólicas de cada especie (Longinelli, 1984; Luz et al., 1984; Kohn, 1996; Kohn et al., 1996; Iacumin y Longinelli, 2002). Por regla general, a mayor temperatura, mayor es la proporción de ${ }^{18} \mathrm{O}$, aunque otros factores, como la fuente de agua evaporada, la cantidad y el tiempo de precipitación o la ingesta de forraje seco, también afectan relación isotópica (McCrea, 1950; Dansgaard, 1964; Gat, 1980; Rozanski et al., 1993; Makarewicz y Pederzani, 2017). En latitudes medias y altas, la composición isotópica de oxígeno del agua meteórica varía estacionalmente, con valores superiores a $\delta^{18} \mathrm{O}$ durante los meses más cálidos (Dansgaard, 1964; Gat, 1980).

En los dientes hipsodontos es posible analizar las variaciones estacionales en las relaciones isotópicas de oxígeno a partir del muestreo secuencial (p. ej., Balasse et al., 2002; Viner et al., 2010; Tornero et al., 2013). En combinación con los isótopos de estroncio, es posible ver si los animales han pastado en diferentes sustratos geológicos en diferentes momentos del año (Bentley y Knipper 2005; Valenzuela-Lamas et al., 2016).

En el caso del ADN antiguo, el principal determinante es la conservación del colágeno y de la hidroxiapatita. Este se suele encontrar muy fragmentado por los procesos tafonómicos y diagenéticos que sufren los restos hasta que son analizados (microorganismos, fluctuaciones en la temperatura, oxidación, hidrólisis, etc.). En nuestra latitud y en yacimientos al aire libre, el grado de éxito de las muestras se sitúa en torno a un tercio de las analizadas. Es decir, de 30 muestras seleccionadas y analizadas, es probable que el ADN esté suficientemente bien conservado en unas diez. El problema se acentúa con materiales que han sido excavados hace tiempo y que se han conservado en sitios con una gran diferencia térmica entre verano e invierno, que es el caso de muchas reservas de materiales arqueológicos. En este sentido, los mejores materiales son los procedentes de cuevas y que han sido excavados recientemente (Geigl, 2002).

El proceso de extracción de ADN antiguo es muy laborioso y costoso, y el riesgo de contaminación hace que solo pueda realizarse en instalaciones específicamente dedicadas a ello. En el caso de los bovinos domésticos y de los cerdos se añade el problema de riesgo de contaminación por los agentes reactivos utilizados si se amplifica por PCR (ver más abajo) y es preciso tomar precauciones adicionales (Champlot et al., 2010). La ventaja de los estudios de $\mathrm{ADN}$ es que, a pesar del riesgo de que funcione solo un número reducido de muestras, es posible ver la llegada de nuevas líneas genéticas y determinar, aproximadamente, el momento de llegada de los nuevos individuos (p. ej., Fernández et al., 2006; Pereira et al., 2009; Verdugo et al., 2019; Frantz et al., 2019).

Los primeros estudios en este campo analizaban el ADN mitocondrial, más abundante que el nuclear, y secuenciaban el ADN mediante la técnica de la clonación (Higuchi et al., 1984). En 1986, la invención de la reacción en cadena de la polimerasa (PCR, por sus siglas en inglés; Mullis et al., 1986) permitió amplificar pequeños fragmentos de ADN y, rápidamente, se aplicó a estudios de ADN antiguo (p. ej., De Salle et al., 1993). Los protocolos 
de extracción, control de la contaminación (ADN endógeno vs. ADN exógeno) y reproducibilidad se fueron mejorando (p. ej., Pruvost et al., 2005; Rohland y Hofreiter, 2007). La invención de la secuenciación de nueva generación en 2005 (NGS, en inglés; Margulies et al., 2005) y, posteriormente, de la técnica de hibridación para enriquecer las muestras (DNA hybridization capture; Briggs et al., 2009) ha permitido reconstruir fragmentos cada vez mayores y hasta secuencias enteras de ADN mitocondrial de diversas especies (Shapiro y Hofreiter 2012; Paijmans et al., 2013). Actualmente, en casos de buena conservación, es posible secuenciar genomas completos, que incluyen el ADN nuclear (p. ej., Fages et al., 2019).

\section{Resultados. Movilidad animal en la península ibérica y el Reino Unido durante la Prehistoria reciente y la época clásica}

\subsection{Morfometría geométrica}

Los estudios de morfometría geométrica con materiales de la península ibérica son todavía escasos, y más aún los centrados en especies domésticas. El estudio de Valenzuela-Lamas et al., 2011 determinó la presencia del ratón doméstico (Mus musculus) y su predominio frente al ratón moruno (Mus spretus) en el siglo IV aC en Alorda Park (Tarragona). Esta especie probablemente llegó a través del comercio marítimo, tal vez desde el área de Cartago, y su abundancia en el registro (un 83\% en un conjunto de 242 mandíbulas) demuestra la ocupación eficaz del nicho ecológico por parte de la forma comensal ya en el siglo IV aC (Valenzuela-Lamas et al., 2011).

Mientras en Francia hay varios estudios sobre bovinos y suinos para explorar los cambios en el tamaño y la identificación de poblaciones y morfotipos durante la prehistoria reciente y época clásica (Nuviala et al., 2014; Duval et al., 2015, 2018), el primer trabajo en la península ibérica ha aparecido hace solo unos pocos meses (Colominas et al., 2019). El estudio revela que las ovejas analizadas del Pirineo y el área del Empordà son morfológicamente diferentes en la Edad del Hierro, mientras que en época romana es probable que haya una mayor circulación de animales entre las dos zonas.

\subsection{Isótopos de estroncio}

Las evidencias sobre movilidad animal a veces se encuentran como elemento secundario en los estudios isotópicos sobre restos humanos, y para la península ibérica los datos son aún escasos (p. ej., Ortega et al., 2013; Díaz Zorita-Bonilla et al., 2017). En el Reino Unido, en cambio, la abundancia de datos publicados permite ofrecer una primera imagen de 


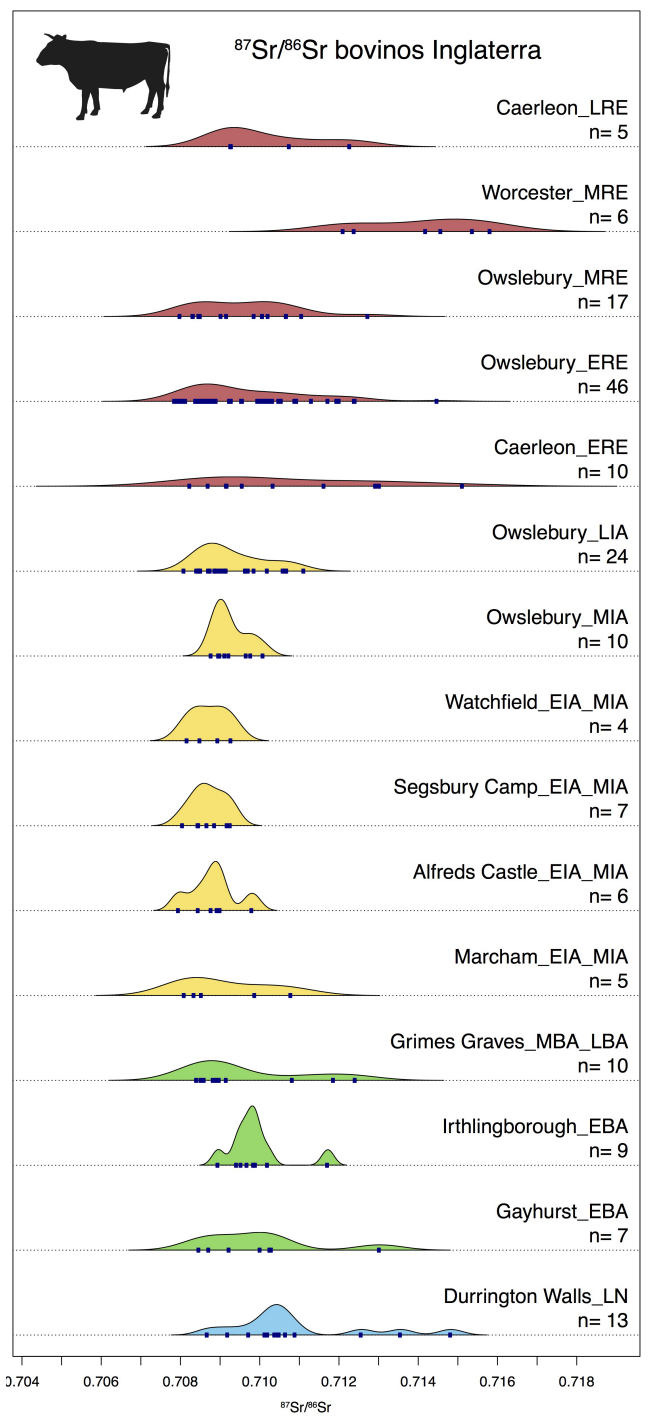

Figura 2. Variabilidad de las ratios isotópicas de estroncio $\left({ }^{87} \mathrm{Sr} /{ }^{86} \mathrm{Sr}\right)$ sobre dientes de bovino de diferentes yacimientos en el Reino Unido (ver localización en la figura 4). Las abreviaturas se refieren a los períodos con sus siglas en inglés. LN: Late Neolithic, EBA: Early Bronze Age, MBA: Middle Bronze Age, LBA: Late Bronze Age, ElA: Early Iron Age, MIA: Middle Iron Age, LIA: Late Iron Age, ERE: Early Roman Empire, MRE: Middle Roman Empire, LRE: Late Roman Empire.

síntesis, aún limitada, de lo que podría ser la movilidad animal en algunos yacimientos del sur de Inglaterra.

Las figuras 2 y 3 muestran las ratios isotópicas de 179 bovinos y 156 cerdos de 14 yacimientos diferentes del sur de Gran Bretaña entre el Neolítico final y época romana. Los yacimientos neolíticos disponibles —Durrington Walls, Mount Pleasant, Marden y West Kennet - corresponden a puntos de encuentro asociados a eventos de banquetes y estructuras monumentales (Viner et al., 2010; Madgwick et al., 2019). Los resultados 
Figura 3. Variabilidad de las ratios isotópicas de estroncio ( ${ }^{87} \mathrm{Sr} /{ }^{86} \mathrm{Sr}$ ) sobre dientes de cerdo de diferentes yacimientos en el Reino Unido. La localización de los yacimientos se encuentra en la figura 4. Las abreviaturas de los períodos cronológicos responden al mismo código que en la figura 2.

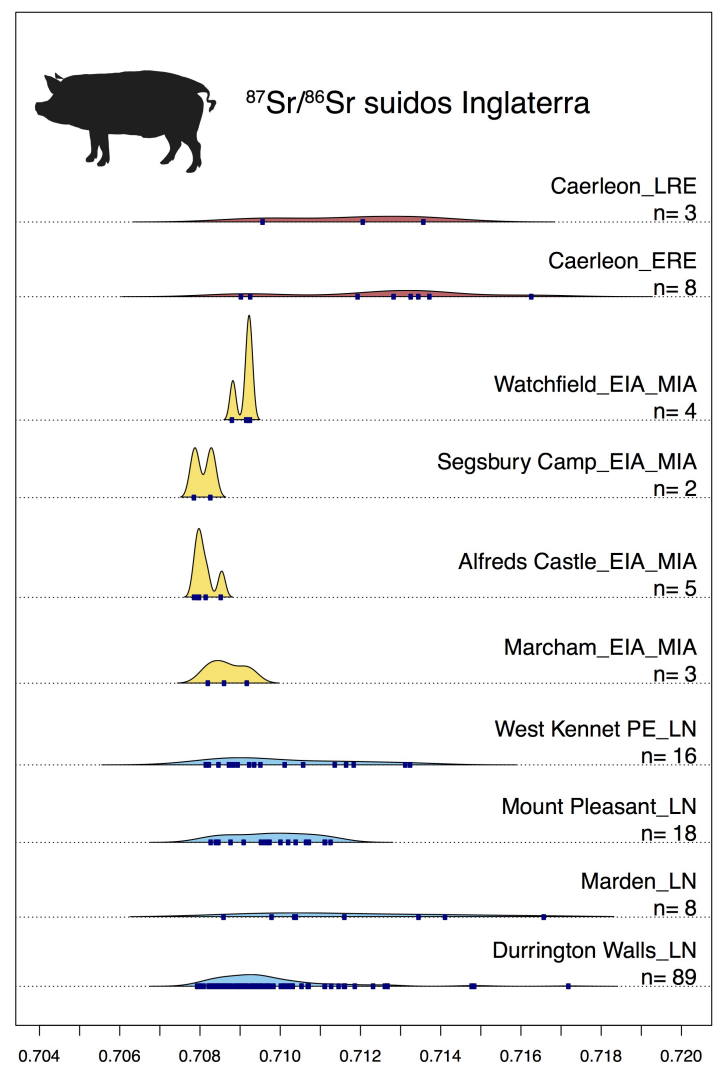

isotópicos de estroncio demuestran que a estos yacimientos llegaban animales de geologías muy diversas, en algunos casos distantes más de $100 \mathrm{~km}$ del yacimiento, incluso en el caso de los cerdos (Viner et al., 2010; Madgwick et al., 2019; figura 4). En lo tocante a los yacimientos del bronce antiguo de Gayhurst e Irthlingborough, ambos corresponden a depósitos de cientos de animales (mayoritariamente bovinos) asociados a tumbas de un único individuo masculino (Towers et al., 2010). Los resultados de estroncio evidencian animales de varias procedencias geológicas, mientras que en el yacimiento de hábitat de Grimes Graves, datado entre el bronce medio y el bronce final, se documentan algunos animales de procedencia lejana, a más de $150 \mathrm{~km}$ del yacimiento (Towers et al., 2017). En la primera y segunda Edad del Hierro (800-200 aC), al yacimiento ritual de Marcham también llegan animales de distintas procedencias, mientras que en los yacimientos de hábitat fortificados - Segsbury, Alfred's Castle, Watchfield y Owslebury - el rango de diversidad es mucho menor. Owslebury, en la fase final de la Edad del Hierro (siglo i aC y primera mitad del siglo I dC) presenta ratios de estroncio más diversas, y esto se acentúa significa- 
tivamente en época romana, cuando el yacimiento era un asentamiento rural cercano a la ciudad de Winchester (Minniti et al., 2014). También en época romana, el campamento militar de Caerleon y el mercado de Worcester presentan una diversidad notable de procedencias de bovinos y cerdos (Madgwick et al., 2019b; Min Gan et al., 2018), que coincide con la circulación a larga distancia de otros materiales (p. ej., vino, aceite, cerámica, etc.).

Así, se documenta una mayor diversidad de ratios de estroncio (y, por tanto, una mayor diversidad de orígenes geográficos de los animales) en los conjuntos del Neolítico y época romana, mientras que los yacimientos datados en la Edad del Hierro (EIA, MIA, LIA) presentan una menor diversidad, tanto en bovinos como en cerdos. Una primera conclusión rápida pudiera ser que había mayor movilidad de animales durante el Neolítico y época romana. Pero antes de hacer inferencias se deben tener en cuenta varios aspectos. En primer lugar, cada yacimiento debe ser analizado según su localización (y, por tanto, su variabilidad local de estroncio, figura 4), así como su funcionalidad. En este sentido, la mayoría de yacimientos tiene una variabilidad geológica similar, lo que facilita la comparación entre ellos. En cambio, Caerleon y Worcester tienen una mayor diversidad geológica en sus inmediaciones: en estos casos, es difícil determinar si la variabilidad observada se debe a la localización de los yacimientos —en una área con una notable diversidad geológica- o bien refleja una particularidad de época romana. En relación con la funcionalidad, todos los conjuntos neolíticos corresponden a puntos de encuentro, mientras que los yacimientos de la Edad del Hierro son mayoritariamente lugares de hábitat. Potencialmente, esto pudiera explicar las diferencias observadas entre los dos períodos. De acuerdo con esto, el yacimiento ritual de Marcham presenta una mayor diversidad que el resto de conjuntos de la Edad del Hierro, lo que apoyaría la idea que la funcionalidad del yacimiento es clave para caracterizar y entender la variabilidad. A la hora de realizar comparaciones diacrónicas, es mejor disponer de yacimientos con una función similar. En este caso, Owslebury constituye un buen caso de estudio, ya que fue un yacimiento de hábitat en los dos períodos (fortificado en la fase de MIA, sin fortificación en la fase de LIA, y núcleo de producción rural en época romana). En este caso, se observa una mayor movilidad de bovinos en época romana, y los resultados estadísticos indican que los cambios entre períodos son significativos (Minniti et al., 2014). Así pues, parece existir una correlación entre la funcionalidad de los yacimientos y el grado de variabilidad de los isótopos de estroncio (mayor en los yacimientos rituales, menor en los yacimientos de hábitat), así como, posiblemente, variaciones diacrónicas.

En el caso de la península ibérica, la diversidad geológica y el escaso número de estudios sobre movilidad animal —que contrasta con el número de estudios disponibles sobre movilidad humana- no permiten ofrecer una imagen de síntesis. En el caso de los yacimientos portugueses de Perdigões, Zambujal y Leceia (Neolítico y Calcolítico; Žalaitè et al., 2018, Wright et al., 2019) se documenta la presencia de algunos animales de procedencias diversas, no compatibles con las ratios de estroncio documentadas en las inmediaciones de los yacimientos. Este es también el caso de algunos équidos de Can Roqueta del Bronce final y la primera Edad del Hierro (Albizuri et al., 2019). Esto contrasta con los 35 caprinos analizados (ovejas y cabras) del yacimiento íbero del Turó de la Font de la Canya (Valenzuela-Lamas et 


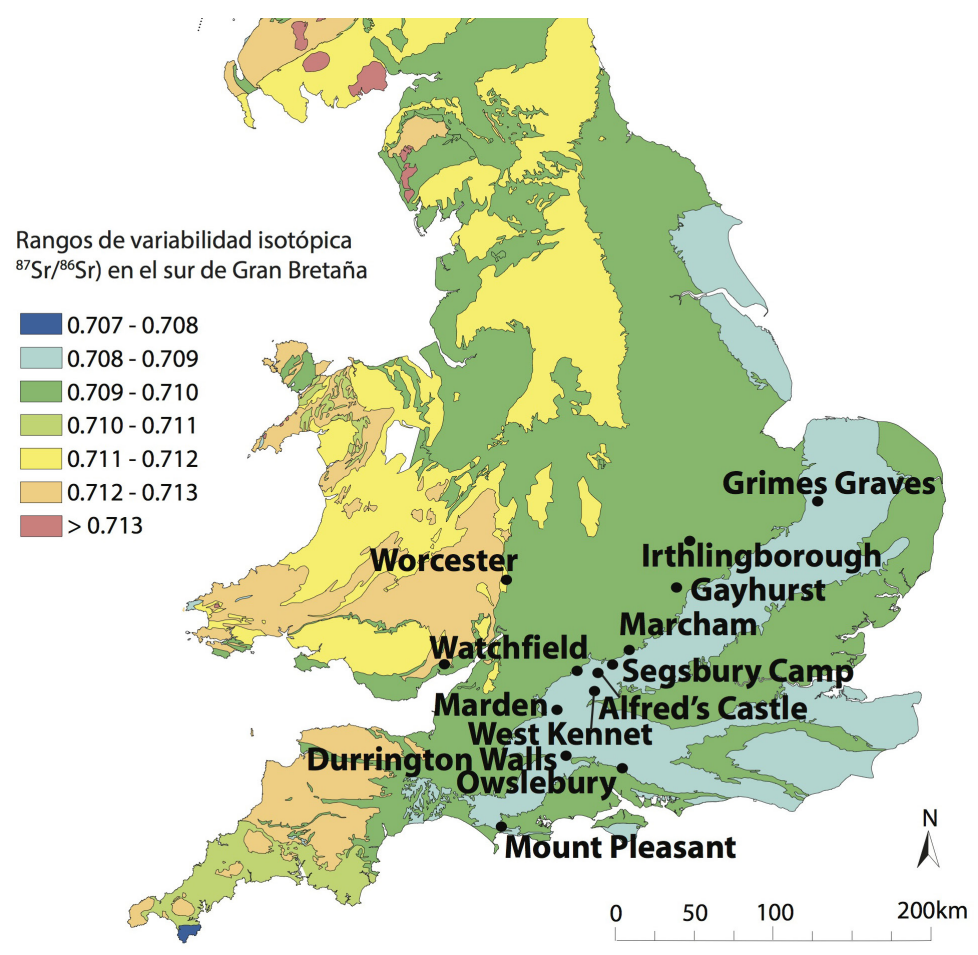

Figura 4. Mapa de estroncio (a partir de Evans et al., 2010) y localización de los yacimientos de las figuras 2 y 3.

al., 2016; 2018): a pesar de la variabilidad isotópica potencial de esta zona, la gran mayoría de ovejas y cabras tenían ratios de estroncio compatibles con la geología presente en las inmediaciones del yacimiento. Esto sugiere que diferentes especies pueden tener diferentes patrones de movilidad. En este sentido, es significativo que una especie de elevado prestigio y carácter simbólico (el caballo), tenga una mayor movilidad que la especie más habitual en los conjuntos de la Edad del Hierro de esta zona (las ovejas y las cabras). Es necesario disponer de más estudios, tanto en lo diacrónico como en diferentes yacimientos del mismo período y con especies diferentes, para poder entender la movilidad animal en toda su complejidad.

\subsection{Isótopos de oxígeno}

Al igual que sucede con los isótopos de estroncio, los estudios sobre movilidad animal en la península ibérica basados en isótopos de oxígeno escasean. El estudio de Waterman et al. (2016) documenta la presencia de cambios significativos en la composición isotópica de oxígeno de las ovejas de Zambujal entre la fase del III milenio aC y la de la primera 
mitad del II milenio aC. En el trabajo se barajan diferentes posibilidades, entre ellas un cambio de las condiciones ambientales, que obligaron a cambiar la zona de pasto, o tal vez diferentes patrones de movilidad o abastecimiento entre los dos períodos cronológicos. Un segundo ejemplo es el estudio de Valenzuela-Lamas et al. (2016), donde se publicaban los resultados de un estudio piloto sobre tres dientes de oveja de la Edad del Hierro combinando isótopos de estroncio y oxígeno. En este caso particular no se detectó ningún movimiento estacional en los animales analizados, ya que las ratios isotópicas de estroncio eran similares en diferentes momentos del año. El estudio piloto de Tornero et al. (2018), basado en dos ovejas trashumantes actuales, ofrece un punto de partida para entender la variación esperada cuando una oveja se mueve del llano a un ecosistema de montaña (> 1800 m.s.n.m) así como la existencia de variabilidad interindividual. Se hace, pues, necesario disponer de más estudios para poder profundizar en la materia, aunque varios trabajos advierten de la existencia de múltiples factores que pueden afectar las ratios isotópicas de oxígeno (p. ej., Makarewicz y Pederzani, 2017).

\subsection{ADN antiguo}

En contraste con la morfometría geométrica y los estudios isotópicos de movilidad, los estudios de ADN antiguo sobre animales de la Prehistoria reciente de la península ibérica son más abundantes. Ya en 2005, Anderung et al. determinaron la presencia del haplotipo Tl — característico de los bovinos del Norte de África- en bovinos de la península ibérica en la Edad del Bronce, y el estudio de Colominas et al. (2015) permitió confirmar la presencia del haplotipo Tl desde el Neolítico en La Draga (Banyoles), así como una mayor diversidad de haplotipos en época romana, todos del tipo T. El estudio de Beja-Pereira et al. (2006), con numerosas muestras de la península ibérica, también sugiere una fuerte influencia del Norte de África en las líneas genéticas actuales de bovinos de la Península. Los autores afirman que, probablemente durante el Neolítico, los bovinos domesticados en Oriente Próximo se cruzaron con uros de diferentes puntos - entre los cuales los del Norte de África-y desde allí se propagaron por vía marítima hacia la península ibérica.

En cuanto a los équidos, tradicionalmente los fenicios se habían considerado los introductores del asno doméstico (Equus asinus; Altuna, 1980; Roselló y Morales, 1994; Nadal et al., 2010). En 2017, el estudio de ADN sobre un diente de équido recuperado en Leceia (Portugal) y datado en el Calcolítico, permitió documentar la presencia de asno mucho antes de la fundación de las colonias fenicias en el sur de la península ibérica (Cardoso et al., 2017). Los autores del estudio apuntan al comercio entre el sur de la Península y el Norte de África durante el III milenio aC como probable origen de esta especie en la Península, juntamente con objetos de marfil, también recuperados en Leceia y otros yacimientos calcolíticos de la zona.

Otro de los debates desarrollados a lo largo de varias décadas se refiere a la presencia de un foco de domesticación del caballo (Equus caballus) en la península ibérica (Uerpmann, 
1990; Lira et al., 2010; Warmuth et al., 2011). El estudio más reciente y extenso (Fages et al., 2019) sugiere que los caballos de la Península apenas han incidido en los genomas de caballo actuales, por lo que no puede ser uno de los focos de domesticación.

Los perros (Canis lupus familiaris) también han sido objeto de estudios de ADN antiguo para entender los flujos genéticos y la selección de ciertos caracteres (Frantz et al., 2016; Catagnano, 2016; Pires et al., 2017). En la actualidad, los perros de la península ibérica y el Norte de África tienen una diversidad genética notable, con haplotipos de las ramas A, B, C y D (Pires et al., 2006). En contraste con épocas más antiguas, la época romana es el período con más diversidad genética, solo superada en la actualidad, lo que sugiere una movilidad notable de perros de diferentes zonas geográficas (Pires et al., 2017). Esta mayor movilidad fue acompañada de una mayor diversidad de morfologías en época clásica en relación con otros períodos del pasado, tal como evidencian numerosos estudios biométricos (ver referencias en Pires et al., 2017).

En el caso de los suidos, los estudios de ADN antiguo indican que los cerdos de la península ibérica y, en general, de Europa occidental, no tienen afinidad genética con los jabalíes de Armenia, Turquía y, en general, Oriente Próximo (Larson et al., 2005; 2007; Frantz et al., 2019). Los autores sugieren que hubo una extensa hibridación entre cerdos domésticos y jabalíes locales en diferentes puntos de Europa, lo que dio pie a haplotipos diferenciados en distintos lugares.

Es curioso que, siendo el ganado más abundante en la Prehistoria de la península ibérica (al menos en el Sur y el Levante peninsular), las ovejas y las cabras no hayan sido aún objeto de estudios de ADN antiguo en la Península. Probablemente, en los próximos años se cubrirá este vacío en la investigación.

\section{Muestreo para el futuro: cómo compatibilizar las diferentes técnicas}

Una limitación que tenemos en arqueología es la naturaleza destructiva de algunas técnicas de investigación, desde la excavación hasta los análisis químicos. Esto, juntamente con la escasez de materiales y la naturaleza única de algunas piezas (sobre todo en las cronologías más antiguas), hace necesario que los análisis sean lo menos destructivos posible.

En el caso de los materiales faunísticos, los dientes son el material más preciado por su durabilidad y su carácter diagnóstico, lo que posibilita la aplicación de múltiples técnicas sobre ellos. Por ello, es necesario preservarlos en la medida de lo posible para futuros trabajos.

La figura 5 ofrece una síntesis de las zonas utilizadas en diversos estudios de movilidad y estacionalidad. Potencialmente, hay zonas en las que pudiera haber solapamiento entre diversas técnicas, como la cara mesio-bucal del protocónido (zona B en la figura 5). 


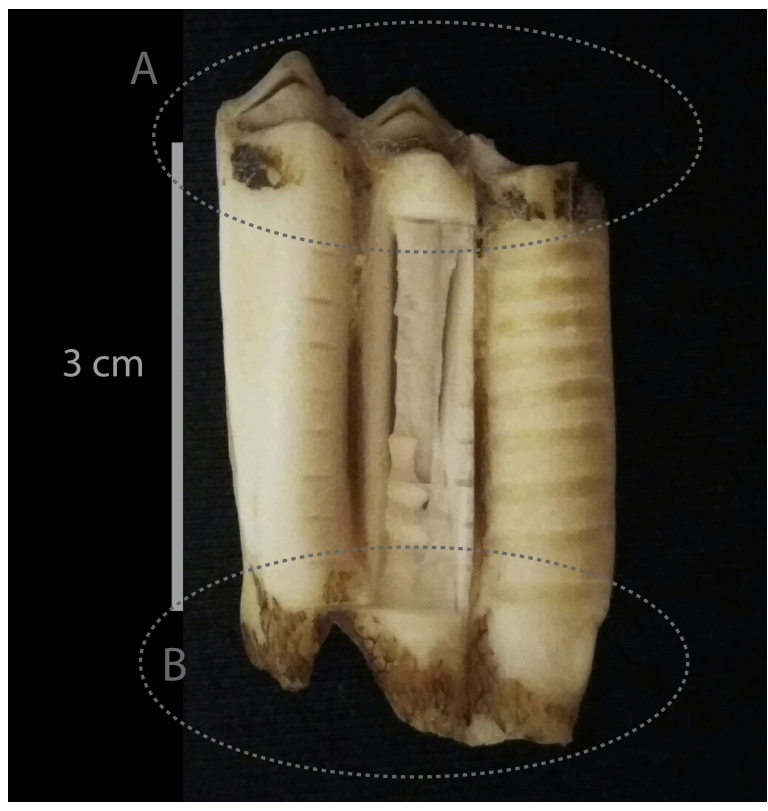

Figura 5. Ejemplo de diferentes técnicas de muestreo para isótopos en una molar inferior de oveja: bandas (derecha), segmentos (centro) y láser en superficie curva (izquierda). La cara oclusal y la base del diente se pueden mantener para otros análisis. Zona A: microdesgaste, morfometría geométrica; zona B: cementocronología, ADN, cálculo dental.

Teniendo en cuenta la variedad de técnicas conocidas hasta ahora, se puede diseñar el muestreo para intentar minimizar el impacto de los análisis destructivos. Por ejemplo, en el caso del muestreo secuencial para análisis isotópicos, se puede optar por muestrear el metacónido o el paracónido en lugar del protocónido, dado que las muestras obtenidas en diferentes cónidos son comparables (Balasse, 2002). Además, el desarrollo de la ablación laser en muestras de perfil curvado para los análisis químicos (Le Roux et al., 2014) —si bien aún en curso y en fase de mejora de protocolos de muestreo y análisis- permitirá mejorar significativamente la integridad de las muestras, al evitar la extracción de pilares enteros de esmalte (p. ej., Lewis et al., 2014), de segmentos (p. ej., Britton et al., 201 1; ValenzuelaLamas et al., 2016) o de bandas transversales (p. ej., Balasse et al., 2002; Zazzo et al., 2012).

Sea cual sea el protocolo de muestreo y la técnica de análisis, el principio de base debe ser reducir lo máximo posible el grado de destrucción de la muestra.

\section{Reflexiones finales y perspectivas de futuro}

Los estudios de movilidad animal tienen un gran potencial para informar de procesos históricos, económicos y modos de gestión del paisaje. A día de hoy, se pueden utilizar múltiples técnicas, ya sea individualmente o en combinación, para reconstruir el grado de movilidad de los animales (también de las poblaciones humanas) y comparar las 
informaciones con otros indicadores arqueológicos (arquitectura, cultura material, datos paleoambientales, etc.).

El carácter destructivo de algunas técnicas constituye una limitación en los análisis a gran escala, tanto por los problemas de conservación del material como por la reproducibilidad de los resultados. En este sentido, la morfometría geométrica es la técnica idónea para la caracterización de morfotipos, ya que no destruye el material. Su principal limitación es la necesidad de un volumen importante de restos, que no siempre se encuentra disponible en el registro arqueológico.

En segundo lugar, los estudios de ADN antiguo generalmente requieren una pequeña muestra, con frecuencia obtenida de la raíz del diente o de huesos largos. En este caso, los puntos críticos son la conservación del colágeno y la hidroxiapatita, así como el elevado coste de los análisis. Los mejores materiales son los procedentes de cuevas, los excavados recientemente y, en general, los que han sufrido menos alteraciones térmicas a lo largo del tiempo. En este sentido, las mejores reservas arqueológicas son las situadas en subterráneos, ya que son los que propician una temperatura más constante a lo largo del tiempo.

En tercer lugar —en orden de cantidad muestra necesaria- se encuentran los isótopos de oxígeno y estroncio, en los que la técnica de muestreo varía enormemente de un estudio a otro, desde ablación láser, muestreo por bandas, muestreo por segmentos, a muestreo de todo un cónido o segmentos muy amplios. La abrasión láser está todavía en desarrollo, pero permitirá mejorar sobre manera la conservación de las muestras, si se solventa la necesidad de tener una superficie plana. Algunos estudios recientes (Le Roux et al., 2014; Willmes et al., 2016) apuntan en ese sentido.

Finalmente, y aunque no la hemos tratado aquí por no encontrar estudios sobre material de la península ibérica, la cementocronología sería la técnica más destructiva a día de hoy, ya que es necesario hacer una lámina fina para observar las curvas de crecimiento del esmalte.

A modo de conclusión, y comparando las diferentes líneas de evidencia disponibles, los trabajos apuntan, consistentemente, que el grado de movilidad animal en época romana fue mayor en comparación con la Edad del Hierro (Minniti et al., 2014; Colominas et al., 2015; Pires et al., 2017; Colominas et al., 2019; Nieto et al., aceptado), y que el rango de movilidad puede depender de la especie analizada (Albizuri et al., 2019; Nieto et al., en prensa).

Tal como hemos propuesto en trabajos anteriores (Valenzuela-Lamas y Albarella, 2017; Albizuri et al., 2019; Nieto et al. en prensa), creemos que los patrones de movilidad animal y las prácticas ganaderas reflejan el nivel de integración económica y la situación sociopolítica de las poblaciones humanas. Se requieren muchos más estudios que permitan caracterizar la movilidad humana y animal en diferentes tipos de yacimientos y cronologías para entender cómo los cambios en la movilidad afectan diferentes aspectos de la vida (p. ej., economía, sociedad). Así mismo, hay diversos retos metodológicos a abordar, como asegurar la comparabilidad entre diferentes prácticas y parámetros de análisis, la comparación entre yacimientos que tienen rangos de variabilidad potencial diferentes, o ampliar el número de referenciales actuales, entre otros. 
En los últimos veinte años se ha avanzado mucho en las diversas técnicas analíticas, y los diferentes campos están en constante búsqueda de mejoras que reduzcan el volumen de muestra necesario y optimicen la comparabilidad y reproducibilidad de los datos. Queremos recalcar la necesidad de que tanto especialistas como responsables de las excavaciones trabajen en colaboración y simbiosis, ya que es fundamental para la buena contextualización e interpretación de los datos en clave arqueológica e histórica.

\section{Agradecimientos}

Al comité de redacción de Pyrenae por proponerme el reto de este artículo. Y a las investigadoras que, a lo largo de los años, han respondido mis insaciables preguntas sobre la aplicación e interpretación de los datos y las mejores técnicas de muestreo: Marie Balasse, Delphine Bosch, Sandi Copeland, Marta Díaz Zorita-Bonilla, Jane Evans, Allowen Evin, Maria Fontanals, Maite-Iris García Collado, Claudia Gerling, Catarina Ginja, Sílvia Guimarães, Maura Pellegrini (espero no olvidarme a nadie). A todos ellos mi agradecimiento y reconocimiento sincero. Este trabajo se ha realizado en el marco del proyecto ERCStarting Grant 716298 ZooMWest - Zooarchaeology and mobility in the Western Mediterranean: from the Late Bronze Age to Late Antiquity. Cualquier omisión o malinterpretación es responsabilidad mía.

\section{Referencias}

ALTUNA, J., 1980, Historia de la domesticación animal en el País Vasco desde sus orígenes hasta la romanización, Sociedad de Ciencias Aranzadi, San Sebastián.

ANDERUNG, C., BOUWMAN, A., PERSSON, P., CARRETERO, J. M., ORTEGA, A.I., ELBURG, R. ... y GÖTHERSTRÖM, A., 2005, Prehistoric contacts over the Straits of Gibraltar indicated by genetic analysis of Iberian Bronze Age cattle, Proceedings of the National Academy of Sciences 102(24), 8431-8435.

BALASSE, M., 2002, Reconstructing dietary and environmental history from enamel isotopic analysis: time resolution of intra-tooth sequential sampling, International Journal of Osteoarchaeology 12(3), 155-165.
BALASSE, M., AMBROSE, S.H., SMITH, A. B. y PRICE, T. D., 2002, The seasonal mobility model for prehistoric herders in the south-western Cape of South Africa assessed by isotopic analysis of sheep tooth enamel, Journal of Archaeological Science 29(9), 917-932.

BENTLEY, R. A. y KNIPPER, C., 2005, Transhumance at the early Neolithic settlement at Vaihingen (Germany), Antiquity 79(306), 1-3.

BOCHERENS, H., KOCH, P. L., MARIOTTI, A., GERAADS, D., Y JAEGER, J. J., 1996, Isotopic biogeochemistry $\left({ }^{13} \mathrm{C},{ }^{18} \mathrm{O}\right)$ of mammalian enamel from African Pleistocene hominid sites, Palaios, 306-318. 
BOOKSTEIN, F. L., 1991, Morphometric tools for landmark data: geometry and biology, Cambridge University Press, Cambridge.

BRIGGS, A. W., GOOD, J. M., GREEN, R. E., KRAUSE, J., MARICIC, T., STENZEL, U., LALUEZA-FOX, C., RUDAN, P., BRAJKOVIC, D., KUCAN, Ž., GUŠIC , I., SCHMITZ, R., DORONICHEV, V. B., GOLOVANOVA, L. V., DE LA RASILLA, M., FORTEA, J., ROSAS, A. Y PÄÄBO, S., 2009, Targeted Retrieval and Analysis of Five Neandertal mtDNA Genomes, Science 325, 318-321.

BRITTON, K., GRIMES, V., DAU, J. y RICHARDS, M. P., 2009, Reconstructing faunal migrations using intra-tooth sampling and strontium and oxygen isotope analyses: a case study of modern caribou (Rangifer tarandus granti), Journal of Archaeological Science 36(5), 1163-1172.

BRITTON, K., GRIMES, V., NIVEN, L., STEELE, T.E., MCPHERRON, S., SORESSI, M. ... Y RICHARDS, M. P., 2011, Strontium isotope evidence for migration in late Pleistocene Rangifer: Implications for Neanderthal hunting strategies at the Middle Palaeolithic site of Jonzac, France, Journal of Human Evolution 61 (2), 176-185.

CARDOSO, J. L., VILSTRUP, J. T., EISENMANN, V. y ORLANDO, L., 2013, First evidence of Equus asinus L., en The Chalcolithic disputes the Phoenicians as the first to introduce donkeys into the Iberian Peninsula, Journal of Archaeological Science 40(12), 4483-4490.

CATAGNANO, V., 2016, Aproximación morfométrica y paleogenética al estudio de la variabilidad de C. 1 . familiaris en la Península Ibérica desde el Neolítico hasta época romana y su contextualización en el ámbito del Mediterráneo occidental. Universitat Autonòma de Barcelona (tesis de doctorado).

CERLING, T. E., HARRIS, J. M., AMBROSE, S. H., LEAKEY, M. G. y SOLOUNIAS, N., 1997, Dietary and environmental reconstruction with stable isotope analyses of herbivore tooth enamel from the Miocene locality of Fort Ternan, Kenya, Journal of Human Evolution 33(6), 635-650.

CHAMPLOT, S., BERTHELOT, C., PRUVOST, M., BENNETT, E. A., GRANGE, T. y GEIGL, E. M.,
2010, An efficient multistrategy DNA decontamination procedure of PCR reagents for hypersensitive PCR applications, PLOS ONE, 5(9), e13042.

COLOMINAS, L., EDWARDS, C. J., BEJAPEREIRA, A., VIGNE, J. D., SILVA, R. M., CASTANYER, P., ... y HOWE, C. J., 2015 , Detecting the $\mathrm{Tl}$ cattle haplogroup in the Iberian Peninsula from Neolithic to medieval times: new clues to continuous cattle migration through time, Journal of Archaeological Science 59, 110-117.

COLOMINAS, L., EVIN, A., BURCH, J., CAMPMAJÓ, P., CASAS, J., CASTANYER, P., ... Y TREMOLEDA, J., 2019, Behind the steps of ancient sheep mobility in Iberia: new insights from a geometric morphometric approach, Archaeological and Anthropological Sciences, 1-12.

CUCCHI, T., VIGNE, J. D. y AUFFRAY, J. C., 2005, First occurrence of the house mouse (Mus musculus domesticus Schwarz \& Schwarz, 1943) in the Western Mediterranean: a zooarchaeological revision of subfossil occurrences, Biological Journal of the Linnean Society 84(3), 429-445.

DAVIS, S. J., SVENSSON, E. M., ALBARELLA, U., DETRY, C., GÖTHERSTRÖM, A., PIRES, A. E. y GINJA, C., 2012, Molecular and osteometric sexing of cattle metacarpals: a case study from $15^{\text {th }}$ century AD Beja, Portugal, Journal of Archaeological Science 39(5), 1445-1454.

DESALLE, R., BARCIA, M. y WRAY, C., 1993, PCR jumping in clones of 30-million-year-oldDNA fragments from amber preserved termites (Mastotermes electrodominicus), Experientia 49, 906-909.

DÍAZ ZORITA-BONILLA, M., KNUDSON, K. J., ESCUDERO CARRILLO, J., BOCHERENS, H. Y GARCÍA SANJUÁN, L., 2017, Mobility patterns and paleodietary insights into humans and cattle at the Copper Age Mega-site of Valencina (Seville, Spain) through ${ }^{18} \mathrm{O}$ and ${ }^{13} \mathrm{C}$ isotope analyses, Menga: Revista de prehistoria de Andalucía 8, 53-68.

DUVAL, C., LEPETZ, S., HORARD-HERBIN, M. P. y CUCCHI, T., 2015, Did Romanization impact Gallic pig morphology? New insights from molar geometric morphometrics, Journal of Archaeological Science 57, 345-354. 
EVANS, J. A., MONTGOMERY, J., WILDMAN, G. y BOULTON, N., 2010, Spatial variations in biosphere 87Sr/86Sr in Britain, Journal of the Geological Society, 167(1), 1-4.

EVIN, A., DOBNEY, K., SCHAFBERG, R., OWEN, J., VIDARSDOTTIR, U. S., LARSON, G. Y CUCCHI, T., 2015, Phenotype and animal domestication: A study of dental variation between domestic, wild, captive, hybrid and insular Sus scrofa, $B M C$ Evolutionary Biology 15(1), 6.

EVIN, A., DOBNEY, K. y CUCCHI, T., 2017, A history of pig domestication: new ways of exploring a complex process, Ecology, Conservation and Management of Wild Pigs and Peccaries. Cambridge University Press, Cambridge.

FAGES, A., HANGHØJ, K., KHAN, N., GAUNITZ, C., SEGUIN-ORLANDO, A., LEONARDI, M., ... y ALFARHAN, A. H., 2019 , Tracking five millennia of horse management with extensive ancient genome time series, Cell 177(6), 1419-1435.

FERNÁNDEZ, H., HUGHES, S., VIGNE, J. D., HELMER, D., HODGINS, G., MIQUEL, C., ... y TABERLET, P., 2006, Divergent mtDNA lineages of goats in an Early Neolithic site, far from the initial domestication areas, Proceedings of the National Academy of Sciences 103(42), 15375-15379.

FRANTZ, L. A. F., MULLIN, V. E., PIONNIERCAPITAN, M., LEBRASSEUR, O., OLLIVIER, M., PERRI, A., LINDERHOLM, A., MATTIANGELI, V., TEASDALE, M. D., DIMOPOULOS, E. A.,

TRESSET, A., DUFFRAISSE, M., MCCORMICK, F., BARTOSIEWICZ, L., GÁL, E., NYERGES, E. A., SABLIN, M. V., BRÉHARD, S., MASHKOUR, M., BALASESCU, A., GILLET, B., HUGHES, S., CHASSAING, O., HITTE, C., VIGNE, J. D., DOBNEY, K., HANNI, C., BRADLEY, D. G. Y LARSON, G., 2016, Genomic and archaeological evidence suggest a dual origin of domestic dogs, Science 352, 1228e1231, <https://doi.org/10.1126/ science.aaf3161>.

FRANTZ, L. A., HAILE, J., LIN, A. T., SCHEU, A., GEÖRG, C., BENECKE, N., ... y BATTISTA, V. M., 2019, Ancient pigs reveal a near-complete genomic turnover following their introduction to Europe, Proceedings of the National Academy of Sciences 116(35), 17231-17238.

GEIGL, E. M., 2002, On the circumstances surrounding the preservation and analysis of very old DNA, Archaeometry 44(3), 337-342.

GERLING, C., DOPPLER, T., HEYD, V., KNIPPER, C., KUHN, T., LEHMANN, M. F., ... y SCHIBLER, J., 2017, High-resolution isotopic evidence of specialised cattle herding in the European Neolithic, PLOS ONE 12(7), e0180164.

HIGUCHI, R., BOWMAN, B., FREIBERGER, M., RYDER, O. A. Y WILSON, A. C., 1984, DNA sequences from the quagga, an extinct membre of the horse family, Nature 312, 282-284.

HOPPE, K. A., STOVER, S. M., PASCOE, J. R. y AMUNDSON, R., 2004, Tooth enamel biomineralization in extant horses: implications for isotopic microsampling, Palaeogeography, Palaeoclimatology, Palaeoecology 206(3-4), 355-365.

KRISTIANSEN, K., 2014, Towards a new paradigm. The third science revolution and its possible consequences in archaeology, Current Swedish Archaeology 22, 11-34.

LARSON, G., DOBNEY, K., ALBARELLA, U., FANG, M., MATISOO-SMITH, E., ROBINS, J. ... Y ROWLEY-CONWY, P., 2005, Worldwide phylogeography of wild boar reveals multiple centers of pig domestication, Science 307(5715), 1618-1621.

LARSON, G., ALBARELLA, U., DOBNEY, K., ROWLEY-CONWY, P., SCHIBLER, J., TRESSET, A., ... y BĂLĂÇSESCU, A., 2007, Ancient DNA, pig domestication, and the spread of the Neolithic into Europe, Proceedings of the National Academy of Sciences 104(39), 15276-15281.

LE ROUX, P. J., LEE-THORP, J. A., COPELAND, S. R., SPONHEIMER, M. y DE RUITER, D. J., 2014, Strontium isotope analysis of curved tooth enamel surfaces by laserablation multi-collector ICP-MS, Palaeogeography, Palaeoclimatology, Palaeoecology 416, 142-149.

LEWIS, J., COATH, C. D. y PIKE, A. W. G., 2014, An improved protocol for ${ }^{87} \mathrm{Sr} /{ }^{86} \mathrm{Sr}$ by laser ablation multi-collector inductively coupled 
plasma mass spectrometry using oxide reduction and a customised plasma interface, Chemical Geology, 390, 173-181.

LIRA, J., LINDERHOLM, A., OLARIA, C., BRANDSTRÖM DURLING, M., GILBERT, M. T. P., ELLEGREN, H., ... y GÖTHERSTRÖM, A., 2010, Ancient DNA reveals traces of Iberian Neolithic and Bronze Age lineages in modern Iberian horses, Molecular Ecology 19(1), 64-78.

LYCETT, S. J., VON CRAMON-TAUBADEL, N. y GOWLETT, J. A., 2010, A comparative 3D geometric morphometric analysis of Victoria West cores: implications for the origins of Levallois technology, Journal of Archaeological Science 37(5), 1110-1117.

MACHOLÁN, M., 2006, A geometric morphometric analysis of the shape of the first upper molar in mice of the genus Mus (Muridae, Rodentia), Journal of Zoology 270(4), 672-681.

MAKAREWICZ, C. A. y SEALY, J., 2015, Dietary reconstruction, mobility, and the analysis of ancient skeletal tissues: expanding the prospects of stable isotope research in archaeology, Journal of Archaeological Science 56, 146-158.

MAKAREWICZ, C. A. y PEDERZANI, S., 2017, Oxygen $\left(\delta^{18} \mathrm{O}\right)$ and carbon $\left(\delta^{13} \mathrm{C}\right)$ isotopic distinction in sequentially sampled tooth enamel of co-localized wild and domesticated caprines: Complications to establishing seasonality and mobility in herbivores, Palaeogeography, palaeoclimatology, Palaeoecology 485, 1-15.

MARGULIES, M., EGHOLM, M., ALTMAN, W. E., ATTIYA, S., BADER, J. S., BEMBEN, L. A., BERKA, J., BRAVERMAN, M. S., CHEN, Y. J., CHEN, Z. T. et al., 2005, Genome sequencing in microfabricated high-density picolitre reactors, Nature 437, 376-380.

MORALES MUÑIZ, A., CEREIJOPECHARROMAN, M. A., HERNÁNDEZCARRASQUILLA, F. y LIESAU VON LETTOWVORBECK, C., 1995a, Of mice and sparrows: commensal faunas from the Iberian Iron Age in the Duero Valley (Central Spain), International Journal of Osteoarchaeology 5(2), 127-138.
MORALES MUÑIZ, A. M., RIQUELME, J. A. Y LIESAU VON LETTOW-VORBECK, C., 1995b, Dromedaries in antiquity: Iberia and beyond, Antiquity 69(263), 368-375.

MULLIS, K., FALOONA, F., SCHARF, S., SAIKI, R. K., HORN, G. T. y ERLICH, H., 1986, Specific enzymatic amplification of DNA in vitro: the polymerase chain reaction, Cold Spring Harbor Symposia on Quantitative Biology 51, 263-273.

NADAL, J., ALBIZURI, S. y MAROTO, J., 2010, Els orígens del burro domèstic a la Mediterrània i a la Península Ibèrica segons les dades arqueològiques, en La recuperació del burro català. Aspectes culturals i biològics, Centre d'Estudis Comarcals de Banyoles, 37-56.

NIETO ESPINET, A., VALENZUELA-LAMAS, BOSCH, D. y GARDEISEN, A., en prensa, 'Off the market?' Zooarchaeological and strontium isotopic results from Iron Age La Monédière and Lattara (Hérault, France), Journal of Archaeological Science: Reports.

ORTEGA, L. A., GUEDE, I., ZULUAGA, M. C., ALONSO-OLAZABAL, A., MURELAGA, X., NISO, J. ... y QUIRÓS CASTILLO, J. A., 2013, Strontium isotopes of human remains from the San Martín de Dulantzi graveyard (Alegría-Dulantzi, Álava) and population mobility in the Early Middle Ages, Quaternary International 303, 54-63.

OWEN, J., DOBNEY, K., EVIN, A., CUCCHI, T., LARSON, G., y VIDARSDOTTIR, U. S., 2014, The zooarchaeological application of quantifying cranial shape differences in wild boar and domestic pigs (Sus scrofa) using 3D geometric morphometrics, Journal of Archaeological Science 43, 159-167.

PAIJMANS, J. L., GILBERT, M. T. P. y HOFREITER, M., 2013, Mitogenomic analyses from ancient DNA, Molecular phylogenetics and evolution 69(2), 404-416.

PEREIRA, F., QUEIRÓS, S., GUSMÃO, L., NIJMAN, I. J., CUPPEN, E., LENSTRA, J. A. ... Y AMORIM, A., 2009, Tracing the history of goat pastoralism: new clues from mitochondrial and Y chromosome DNA in North Africa, Molecular Biology and Evolution 26(12), 2765-2773. 
PIRES, A. E., OURAGH, L., KALBOUSSI, M., MATOS, J., PETRUCCI-FONSECA, F. Y BRUFORD, M. W., 2006, Mitochondrial DNA sequence variation in Portuguese native dog breeds: diversity and phylogenetic affinities, Journal of Heredity 97(4), 318-330.

PIRES, A. E., DETRY, C., FERNÁNDEZRODRÍGUEZ, C., VALENZUELA-LAMAS, S., ARRUDA, A. M., MAZZORIN, J. D. G. ... y GINJA, C., 2018, Roman dogs from the Iberian Peninsula and the Maghreb-A glimpse into their morphology and genetics, Quaternary International 471, 132-146.

POINAR, H. N., SCHWARZ, C., QI, J., SHAPIRO, B., MACPHEE, R. D. E., BUIGUES, B., TIKHONOV, A., HUSON, D. H., TOMSHO, L. P., AUCH, A., RAMPP, M., MILLER, W. Y SCHUSTER, S. C., 2006, Metagenomics to paleogenomics: Large-scale sequencing of mammoth DNA, Science 311, 392-394.

PÖLLATH, N., SCHAFBERG, R. y PETERS, J., 2019, Astragalar morphology: approaching the cultural trajectories of wild and domestic sheep applying geometric morphometrics, Journal of Archaeological Science: Reports 23, 810-821.

PRUVOST, M., GRANGE, T. y GEIGL, E. M., 2005, Minimizing DNA contamination by using UNGcoupled quantitative real-time PCR on degraded DNA samples: application to ancient DNA studies, Biotechniques 38(4), 569-575.

RIQUELME-CANTAL, J. A., SIMÓNVALLEJO, M. D., PALMQVIST, P. Y CORTÉSSÁNCHEZ, M., 2008, The oldest mongoose of Europe, Journal of Archaeological Science 35(9), 2471-2473.

ROHLAND, N. y HOFREITER, M., 2007, Ancient DNA extraction from bones and teeth, Nature protocols $2(7), 1756$.

ROHLF, F. J. y MARCUS, L. F., 1993, A revolution morphometrics, Trends in ecology $\theta$ evolution 8(4), 129-132.

ROSELLÓ, E. y MORALES, A. (ed.), 1994, Castillo de Doña Blanca. Archaeoenvironmental investigations in the Bay of Cádiz, Spain (750-500 B.C.), BAR International Series, 593, Oxford.
SCHLUMBAUM, A., TURGAY, M. y SCHIBLER, J., 2006, Near East mtDNA haplotype variants in Roman cattle from Augusta Raurica, Switzerland, and in the Swiss Evolene breed, Animal Genetics 37(4), 373-375.

SELDEN, R. Z., PERTTULA, T. K. y O'BRIEN, M. J., 2014, Advances in documentation, digital curation, virtual exhibition, and a test of 3D geometric morphometrics: a case study of the Vanderpool vessels from the ancestral Caddo territory, Advances in Archaeological Practice 2(2), 64-79.

SHAPIRO, B. y HOFREITER, M., 2012, Ancient DNA. Methods and Protocols, Humana Press.

SPONHEIMER, M. y LEE-THORP, J. A., 1999, Oxygen isotopes in enamel carbonate and their ecological significance, Journal of Archaeological Science 26(6), 723-728.

TORNERO, C., AGUILERA, M., FERRIO, J. P., ARCUSA, H., MORENO-GARCÍA, M., GARCIAREIG, S. y ROJO-GUERRA, M., 2018, Vertical sheep mobility along the altitudinal gradient through stable isotope analyses in tooth molar bioapatite, meteoric water and pastures: A reference from the Ebro valley to the Central Pyrenees, Quaternary International 484, 94- 106.

UERPMANN, H. P., 1990, Die Domestikation des Pferdes im Chalkolihikum West-und Mitteleuropas, mit 10 Textabbildungen, Madrider Mitteilungen 31, 109-153.

VALENZUELA-LAMAS, S., JIMÉNEZMANCHÓN, S., EVANS, J., LÓPEZ, D., JORNET, R. y ALBARELLA, U., 2016, Analysis of seasonal mobility of sheep in Iron Age Catalonia (north-eastern Spain) based on strontium and oxygen isotope analysis from tooth enamel: First results, Journal of Archaeological Science: Reports 6, 828-836.

VALENZUELA-LAMAS, S., ORENGO, H. A., BOSCH, D., PELLEGRINI, M., HALSTEAD, P., NIETO-ESPINET, A., TRENTACOSTE, A., JIMÉNEZ-MANCHÓN, S., LÓPEZ-REYES, D. y JORNET-NIELLA, R., 2018, Shipping amphorae and shipping sheep? Livestock mobility in the north-east Iberian peninsula during the Iron Age 
based on strontium isotopic analyses of sheep and goat tooth enamel, PLOS ONe 13(10), e0205283.

VERDUGO, M. P., MULLIN, V. E., SCHEU, A., MATTIANGELI, V., DALY, K. G., DELSER, P. M. ... y HESSE, P., 2019, Ancient cattle genomics, origins, and rapid turnover in the Fertile Crescent, Science 365(6449), 173-176.

WARMUTH, V., ERIKSSON, A., BOWER, M. A., CAÑÓN, J., COTHRAN, G., DISTL, O.,

GLOWATZKI-MULLIS, M. L., HUNT, H., LUÍS, C., DO MAR OOM, M. et al., 2011, European domestic horses originated in two holocene refugia, PLOS ONE 6, el8194.

WILLMES, M., KINSLEY, L., MONCEL, M. H., ARMSTRONG, R. A., AUBERT, M., EGGINS, S. y GRÜN, R., 2016, Improvement of laser ablation in situ micro-analysis to identify diagenetic alteration and measure strontium isotope ratios in fossil human teeth, Journal of Archaeological Science 70, 102-116.

WILLMES, M., BATAILLE, C. P., JAMES, H. F., MOFFAT, I., MCMORROW, L., KINSLEY, L.
... y GRÜN, R., 2018, Mapping of bioavailable strontium isotope ratios in France for archaeological provenance studies, Applied Geochemistry 90, 75-86.

WRIGHT, E., WATERMAN, A. J., PEATE, D. W., KUNST, M., CARDOSO, J. L. y DETRY, C., 2019, Animal mobility in Chalcolithic Portugal: Isotopic analyses of cattle from the sites of Zambujal and Leceia, Journal of Archaeological Science: Reports 24, 804-814.

ŽALAIT , I., MAURER, A. F., GRIMES, V., SILVA, A. M., RIBEIRO, S., SANTOS, J. F. ... y VALERA, A. C., 2018, Diet and mobility of fauna from Late Neolithic-Chalcolithic site of Perdigões, Portugal, Journal of Archaeological Science: Reports $19,674-685$.

ZAZZO, A., BENDREY, R., VELLA, D., MOLONEY, A. P., MONAHAN, F. J. Y SCHMIDT, O., 2012, A refined sampling strategy for intra-tooth stable isotope analysis of mammalian enamel, Geochimica et Cosmochimica Acta 84, 1-13. 